Service social

\title{
Le féminisme dans l'action avec les aînées
}

\section{Lise Hébert}

Volume 35, numéro 1-2, 1986

Recherche - Action - Évaluation

URI : https://id.erudit.org/iderudit/706290ar

DOI : https://doi.org/10.7202/706290ar

Aller au sommaire du numéro

Éditeur(s)

École de service social de l'Université Laval

ISSN

1708-1734 (numérique)

Découvrir la revue

Citer ce document

Hébert, L. (1986). Le féminisme dans l'action avec les aînées. Service social, 35(1-2), 5-11. https://doi.org/10.7202/706290ar d'utilisation que vous pouvez consulter en ligne.

https://apropos.erudit.org/fr/usagers/politique-dutilisation/ 


\section{ÉDITORIAL}

HÉbert, Lise, travailleuse sociale au

Centre de services sociaux de Québec

\section{Le féminisme dans l'action avec les aînées}

Dans cet éditorial, je souhaite partager opinions et réflexions sur l'orientation de l'action en service social avec les aînées. Cette démarche fait suite à près de dix années de pratique avec les personnes âgées au Centre de services sociaux de Québec. La rédaction d'un essai de maîtrise portant sur une expérience que j'ai réalisée avec un groupe de femmes âgées (Hébert, 1985), a contribué à nourrir ces réflexions. Le présent texte s'insère, en quelque sorte, dans un débat élargi, alors que notre société québécoise se confronte au nouveau défi de l'expansion d'une population vieillissante.

Je situerai ce questionnement en exposant d'abord brièvement quelques enjeux socio-politiques actuels, en regard de cette population âgée. Puis, en cherchant à clarifier l'impact de ces décisions politiques, je résumerai comment une vision féministe amène un éclairage différent sur la réalité et les conditions de vie des aînées.

Ceci me permettra d'explorer comment une telle analyse peut influencer l'action à entreprendre avec celles-ci dans les différents secteurs qui ont à composer avec leur réalité. Enfin, ces quelques lignes tenteront de mettre en lumière des éléments de réflexion, en lien avec les politiques actuelles de l'État qui concernent ces femmes et ces hommes avancés en âge.

\section{Le contexte socio-politique}

Au Québec, on assiste actuellement à une modification de l'attitude fondamentale de l'État vis-à-vis les responsabilités qu'il 
assumait durant les années 1960-1970, pour le bien-être de l'ensemble des membres de la société (Duchatel et Vaillancourt, 1979). Avec la crise économique du début des années quatre-vingt et la croissance du taux de la population âgée, soit une hausse de $771 \%$ de 1901 à 1981 au Canada (Conseil national du bien-être social, 1984), l'État est à reviser sa position quant à son rôle auprès de cette population.

De fait, avec cet accroissement, l'État voit aussi augmenter le coût des différents services (par exemple: hébergement en famille d'accueil, pavillons, centres d'accueil, centres hospitaliers pour soins prolongés) qu'il offre aux personnes âgées. Considérant, en outre, cet aspect de la situation, et à la faveur d'une opinion publique qui questionne son type d'intervention, l'État se retire progressivement.

Dans ce contexte où, de plus, de sévères coupures budgétaires sévissent dans les services gouvernementaux (Fortin, 1983), l'État met en œuvre sa politique du vieillissement. Les principes qui y sont énoncés visent à redonner au milieu (bénévolat, famille, voisinage, etc.) et aux personnes âgées elles-mêmes le rôle de répondre davantage aux besoins qu'apporte l'âge avancé (Grandmaison, 1982). Il s'agit donc là d'une politique dite de maintien à domicile des personnes âgées (Ministère des affaires sociales, 1980), où l'État se libère de plus en plus de son rôle de pourvoyeur et vise la prise en charge des besoins de cette population par elle-même et son environnement (Laurin, 1985). Mais un tel changement d'orientation est-il fondé sur une connaissance précise de ce que sont ces personnes âgées et leurs besoins?

\section{Qui sont les personnes âgées?}

Examiner cette situation, c'est d'abord constater que les femmes constituent la majorité des personnes de 65 ans et plus, soit $57,2 \%$ au Canada en 1981 (Conseil national du bien-être social, 1984) ; cette surreprésentation féminine se retrouve également au Québec, avec $57 \%$ de femmes âgées (Dulude, 1980). Sachant que la population âgée de 65 ans et plus s'accroît continuellement et que les femmes ont une espérance de vie supérieure de sept ans à celle des hommes (Conseil national du bien-être social, 1984), il apparaît d'autant plus significatif de considérer spécifiquement la situation de ces femmes. Car cette surreprésentation n'indique pas nécessairement qu'elles soient en meilleure position que les hommes. En effet, au plan économique, on note que les aînées se retrouvent majoritairement sous le seuil de pauvreté. Non rémunérées pour leur travail à la maison ou alors à petit salaire, sans régime de retraite (Dulude, 1978), peu scolarisées, pour la plupart dépendantes du salaire du conjoint, nos aînées se retrouvent 
sans le sou et en situation de veuvage plus souvent que les hommes. Ainsi, $80 \%$ des femmes âgées ont des revenus inférieurs à 10000 en 1982, comparativement à $54 \%$ des hommes âgés (Conseil national du bien-être social, 1984). Cette disparité révèle bien, à mon sens, les effets négatifs d'une société fondée sur la division des sexes (Mitchell, 1974).

Quant à leur santé, elle paraît également précaire. Ainsi, au Canada, les femmes âgées de 65 ans et plus totalisent $12.5 \%$ des problèmes de santé mais, pourtant, cette partie de la population compte seulement pour $4,9 \%$ de l'ensemble de la population canadienne (GRAFS, 1983). À cause des accidents domestiques, beaucoup plus nombreux que chez les hommes du même âge, les aînées subissent des limitations fonctionnelles sérieuses pour de longues périodes (Conseil des affaires sociales, 1982). De fait, les femmes s'occupent toujours des travaux ménagers jusqu'à un âge très avancé et sont quatre fois plus sujettes que les hommes à l'ostéoporose (Harel, 1980). À cela s'ajoutent des problèmes parfois liés, de près ou de loin, à la fonction de reproduction, que les femmes âgées ont grandement occupée, ou à la ménopause : phlébites, varices, arthrite, tumeurs malignes et bénignes (GRAFS, 1983). Au Québec, $80 \%$ des femmes âgées présentent de l'obésité ou un surplus de poids, résultat probable d'une mauvaise alimentation liée à la pauvreté, la solitude, la dépression (GRAFS, 1983). La consommation de médicaments est reconnue comme trop élevée chez les personnes âgées, et elle l'est particulièrement chez les femmes. Ainsi, analgésiques et psychotropes leur sont les plus prescrits (Conseil des affaires sociales, 1982); notons que ces médicaments ont des effets secondaires inquiétants : fatigue, atonie, interférence dans le jugement, qui peuvent aggraver des états dépressifs préexistants (GRAFS, 1983).

De fait, au Québec, les femmes âgées consultent plus que les hommes pour des problèmes d'ordre psychologique (par exemple: la dépression). La condition critique de ces femmes ne semble plus l'effet du hasard. Pour la plupart, elles ont tenu un rôle socialement peu valorisé (mère, épouse) qu'elles perdent en vieillissant. Elles furent dépendantes d'un conjoint pourvoyeur, parfois victimes de violence familiale même lorsqu'âgées (Radio-Canada, 1986). De plus, conditionnées toute leur vie, à des degrés plus ou moins importants, par une religion qui préconisait la soumission de la femme (Chabot et Dunnigan, 1980), et ayant évolué dans une société dont les lois rendaient effective cette dépendance à l'homme (Lachance, 1982), les femmes âgées s'estiment et s'affirment bien difficilement. Ayant intégré cette image négative de la femme, elles subissent, en avançant en âge, le préjugé de la vieillesse laide et inutile (Dulude, 1978). 
La vieillesse n'est donc plus le fait d'une population homogène et asexuée; c'est plutôt majoritairement la situation des femmes alors seules, paurres, passives, souffrantes dans leur corps et leur esprit. Connaissant avec toujours plus d'acuité les méfaits d'une organisation sociale sexiste et stéréotypée, les décisions et les actions ne pourraientelles pas être mieux adaptées à cette réalité ?

\section{Des avenues d'action à explorer}

Ainsi, lorsqu'on constate que la clientèle féminine âgée de 65 ans et plus est majoritaire au Centre de services sociaux de Québec (données opérationnelles) comme au Centre de services sociaux du Montréal Métropolitain (Robichaud, 1983) et, on peut facilement le supposer, pour la plupart des centres locaux de services communautaires au Québec, il apparaît d'autant plus important d'adopter une vision féministe des problèmes rencontrés par cette population à risque.

Déjà des groupes de femmes, des groupes pour la défense des droits des retraités et des préretraités (A.Q.D.R., 1977), des recherches et plusieurs écrits sur le sujet (Dulude, 1978; Harel, 1980 ; GRAFS, 1983) démontrent cette nécessité d'adapter l'intervention à la réalité des femmes (Corbeil et al., 1983), y compris les aînées.

Des décisions de l'État voient timidement le jour pour certains problèmes spécifiques aux femmes (par exemple: intervention féministe et femmes violentées, femmes et santé mentale). Ces efforts devraient se développer davantage, de façon globale, pour atteindre aussi les aînées et leurs conditions de vie. Une volonté politique exprimée en ce sens par le Ministère de la santé et des services sociaux, viendrait consolider les différents programmes d'action dans ce domaine.

Une programmation des services sociaux au niveau des ressources alternatives, ainsi que des centres de services sociaux et des centres locaux de services communautaires, qui tiendrait compte de cette réalité spécifique des femmes avancées en âge, favoriserait l'éclosion de modes d'intervention toujours de mieux en mieux adaptés au vécu de ces femmes. Ainsi, des réseaux de services professionnels ou d'entraide qui élaboreraient leurs actions (individuellement, en groupe ou au plan communautaire) selon une perspective féministe favoriseraient fort possiblement une redéfinition et une revalorisation de cette image des femmes vieillissantes. L'intervention féministe en soi ne se veut pas une réponse à tous les maux. Elle apparaît toutefois comme une des voies d'action pertinentes à développer, pour cette partie de la population qui subit durement les conséquences du sexisme. 
À cet égard, quoique malheureusement peu connus, des tentatives et des projets d'intervention féministe avec des femmes âgées (Hébert, 1985) ont vu le jour dans différents milieux, que ce soit individuellement ou en groupe. Pour supporter ces efforts d'intervenantes sensibilisées à la question et systématiser cette démarche, il semble nécessaire que les milieux académiques et la recherche se penchent sérieusement sur le développement spécifique de ce type d'approche. À tous ces niveaux, de telles préoccupations ne pourraient que déboucher sur une action de plus en plus concertée et adaptée, autant pour ces aînées d'aujourd'hui que pour le grand nombre que nous serons dans les prochaines décennies. Qui sait, ce mouvement aura-t-il un effet d'entrainement chez les hommes pour que leur vieillesse soit aussi plus satisfaisante?

\section{Conclusion}

Finalement, dans ce contexte où l'État décide de prendre de nouvelles orientations, au plan des services accordés aux personnes âgées, je crois donc nécessaire de soulever cette question des conditions de vie des aînées.

On l'a vu, être femme âgée c'est vivre, plus que les hommes, et par l'effet d'une organisation sociale, économique et politique empreinte de sexisme, la pauvreté, le veuvage, la solitude et l'isolement, la dépendance et les limitations dans leur santé physique et mentale. II devient donc primordial que tous les niveaux de décision et d'action s'appuient sur cette réalité. De fait, une question se pose : un discours prônant l'autonomie des personnes âgées, dans le contexte sociopolitique actuel, ne risque-t-il pas de servir d'abord une volonté économique de réduire un budget difficile à équilibrer?

Nous nous devons d'exercer une vigilance car, d'une fin que devrait être l'autonomie des personnes, on risque d'en faire un moyen pour résorber une crise économique. Une autonomie créée de la sorte risque, à mon sens, d'être bien précaire, sinon de ne pas respecter leurs conditions propres et de faire fausse route.

Ces quelques lignes ne prétendent pas faire un tour complet de la question, ni trouver toutes les solutions, mais se voulaient un partage de réflexions qui, s'ajoutant à d'autres, cherchent des voies d'action qui aident les femmes aînées à prendre la place qui leur revient. 


\section{Références bibliographiques}

Association québécoise pour la défense des droits des retraités et des préretraités (A.Q.D.R.), Manifeste: la situation économique des retraités, Montréal, Faculté de l'éducation permanente, Université de Montréal, 1977, 62p.

Chabot, Marc et Lise Dunnigan, Condition féminine - condition masculine : thème religion, recueil de textes du cours SOC-1001, Québec, TéléUniversité, Université du Québec, 1980 : 151-157.

Conseil des affaires sociales et de la famille, Médicaments ou potions magiques?, Québec, Ministère des affaires sociales, 1982, 95p.

Conseil national du bien-être social, Soixante-cinq ans et plus, Ottawa, Ministère des approvisionnements et services, 1984, 83p.

CORBEIL, Christine et al., L'intervention féministe, l'alternative des femmes au sexisme en thérapie, Montréal, Albert Saint-Martin, 1983, 188p.

Duchatel, Jules et Yves Vaillancourt, "Gestion de la crise, politique sociale : stratégie en gestation... ", Compte-rendu du colloque sur les crises et les travailleurs, Montréal, Service des communications, Université du Québec à Montréal, octobre 1979: 107-120.

DULUDE, Louise, "La condition de la femme âgée au Québec", La recherche gérontologique au Québec, Montréal, Les cahiers de l'ACFAS, n 5, 1980 : 91-102.

Dutude, Louise, Vieillir au féminin, Ottawa, Conseil consultatif de la situation de la femme, 1978, 150p.

FORTIN, Denis, "Lutte contre l'injustice et nouvelle solidarité", Service social, vol. $32, n^{\circ} 3,1983$ : 462-465.

GRAFS, Nous, notre santé, nos pouvoirs, Montréal, Albert Saint-Martin, 1983, $202 p$.

Grandmaison, Aline, "La désinstitutionnalisation en regard de la population âgée : un phénomène paradoxal ", Intervention, n 64, 1982: 12-20.

Harel, Louise, Vieillir au féminin, Montréal, Centre de services sociaux du Montréal Métropolitain, 1980, 10p.

HÉBERT, Lise, L'utilisation complémentaire de l'intervention féministe et du modèle de réciprocité dans un groupe de femmes aînées, essai présenté à l'École de service social, même condition à l'obtention du diplôme de maîtrise, Québec, Université Laval, 1985, 95p.

LACHANCE, Lise, "L'histoire des femmes au Québec (2). La défaite des anciens droits : 1832-1900", Le Soleil, 28 décembre 1982.

LAURIN, Camille, "Discours au colloque de l'Association québécoise de gérontologie, 8 novembre 1984 ", Cahiers de l'ACFAS, n 34, 1985 : 10-19.

Ministère des affaires sociales, Pour mieux vieillir au Québec, Québec, document de consultation sur une politique du troisième âge, Gouvernement du Québec, juin 1980, 44p. 
Mitchell, Juliet, L'âge des femmes, Paris, Éditions des femmes, 1974. Voir' en particulier le quatrième chapitre: "La politique du mouvement de libération des femmes".

RADIO-CANADA, documentaire sur: La violence faite par les enfants aux parents âgés, "Le point", deux émissions diffusées les 27 et 28 février 1986.

RoBichaUD, Jean-Bernard, "La composition socio-économique de la clientèle des services sociaux ", Service social, vol. 32, n 3, $1983: 339-354$. 